\title{
Correction to: Geometry and topology of the Kerr photon region in the phase space
}

\author{
Carla Cederbaum $^{1}$ (D) Sophia Jahns ${ }^{1}$ \\ Published online: 20 November 2019 \\ (c) Springer Science+Business Media, LLC, part of Springer Nature 2019
}

\section{Correction to: General Relativity and Gravitation (2019) 51:79 https://doi.org/10.1007/s10714-019-2561-y}

The proof of Theorem 10 can be considerably simplified, as was pointed out to us by Gregory J. Galloway: Indeed, it is unnecessary to rule out $L(2 ; 0)$; since 0 and 2 are not coprime, this case does not need to be considered, which makes it superfluous to argue that $P_{0}$ admits a Seifert fibration without exceptional fibers (Proposition 15). Thus, one is left with the case $P_{0} \approx L(2 ; 1) \approx S O(3)$.

From a slightly different point of view, one may also argue as follows: Since $P_{0}$ is a closed 3-dimensional manifold with $\pi_{1}\left(P_{0}\right)=\mathbb{Z}_{2}$, it is doubly covered by an $\mathbb{S}^{3}$ (by the Poincaré conjecture). By the elliptization conjecture, this $\mathbb{S}^{3}$ can be taken to be the standard 3-sphere and the group $\mathbb{Z}_{2}$ as a subgroup of $S O(3)$ acting on it. (For the statements of the Poincare and the elliptization conjecture, see [4,5]; for the proofs covering these conjectures see [1-3].) Hence, $P_{0}$ is the quotient $\mathbb{S}^{3} / \mathbb{Z}_{2} \approx \mathbb{R} P^{3} \approx$ $S O(3)$.

Recalling how $P_{0}$ was obtained as a slice $P \cap\left\{t=0, p_{0}=-1\right\}$ of the photon region in the phase space, this proves Theorem 10.

\section{References}

1. Perelman, G.: The entropy formula for the Ricci flow and its geometric applications (2002)

2. Perelman, G.: Finite extinction time for the solutions to the Ricci flow on certain three-manifolds (2003)

3. Perelman, G.: Ricci flow with surgery on three-manifolds (2003)

The original article can be found online at https://doi.org/10.1007/s10714-019-2561-y.

Sophia Jahns

jahns@math.uni-tuebingen.de

Carla Cederbaum

cederbaum@math.uni-tuebingen.de

http://math.uni-tuebingen.de/cederbaum

1 University of Tübingen, Tübingen, Germany 
4. Thurston, W.: The Geometry and Topology of Three-Manifolds. Princeton lecture notes on geometric structures on 3-manifolds (1980)

5. Scott, P.: The geometries of 3-manifolds. Bull. Lond. Math. Soc. 15(5), 401-487 (1983)

Publisher's Note Springer Nature remains neutral with regard to jurisdictional claims in published maps and institutional affiliations. 